\title{
Upgrading electron temperature and electron density diagnostic diagrams of forbidden line emission (Research Note)
}

\author{
B. Proxauf ${ }^{1}$, S. Öttl ${ }^{1}$, and S. Kimeswenger ${ }^{2,1}$ \\ ${ }^{1}$ Institute for Astro- and Particle Physics, Leopold Franzens Universität Innsbruck, Technikerstrasse 25, 6020 Innsbruck, Austria \\ e-mail: Bastian.Proxauf@student.uibk.ac.at, Silvia.0ettl@uibk.ac.at \\ 2 Instituto de Astronomía, Universidad Católica del Norte, Avenida Angamos 0610, Antofagasta, Chile \\ e-mail: Stefan.Kimeswenger@gmail.com
}

Received 31 August 2013 / Accepted 20 November 2013

\section{ABSTRACT}

\begin{abstract}
Context. Diagnostic diagrams of forbidden lines have been a useful tool for observers for many decades now. They are used to obtain information on the basic physical properties of thin gaseous nebulae. Some diagnostic diagrams are in wavelength domains that were difficult to apply either due to missing wavelength coverage or the low resolution of older spectrographs. Furthermore, most of the diagrams were calculated using just the species involved as a single atom gas, although several are affected by well-known fluorescence mechanisms as well. Additionally, the atomic data have improved up to the present time.

Aims. The aim of this work is to recalculate well-known, but also sparsely used, unnoted diagnostics diagrams. The new diagrams provide observers with modern, easy-to-use recipes for determining electron temperature and densities.

Methods. The new diagnostic diagrams were calculated using large grids of parameter space in the photoionization code CLOUDY. For a given basic parameter (e.g., electron density or temperature), the solutions with cooling-heating-equilibrium were chosen to derive the diagnostic diagrams. Empirical numerical functions were fitted to provide formulas usable in, e.g., data reduction pipelines. Results. The resulting diagrams differ significantly from those used up to now and will improve thermodynamic calculations. To our knowledge, detailed, directly applicable fit formulas are given for the first time, leading to the calculation of electron temperature or density from the line ratios.
\end{abstract}

Key words. plasmas - planetary nebulae: general - HII regions - galaxies: active

\section{Introduction}

Diagnostic diagrams of forbidden line emission from astrophysical plasmas are widely accepted as a tool for deriving electron temperature $T_{\mathrm{e}}$ and the (number) density $n_{\mathrm{e}}$. The physics is well understood (see Osterbrock \& Ferland 2006, hereinafter OF06), but the hitherto published diagrams mostly do not take any disturbance of the basic processes by other atoms into account, for instance by fluorescence.

\section{The electron temperature $T_{\mathrm{e}}$ :}

To derive the electron temperature $T_{\mathrm{e}}$, at least two metastable levels above the ground level with different energies are required. Thanks to the change in the populations with $T_{\mathrm{e}}$, the line strengths are a direct indicator of its value. In contrast to the determination of the electron densities described below, the collisional de-excitation must not play a role here. This boundary condition is often overlooked by observers when using the classical diagnostic diagrams in dense environments as well. Typical representatives are [O III] $\lambda \lambda(4958 \AA+5007 \AA) / \lambda 4363 \AA$ using the exponential approximation in OF06 and [Ar III] $\lambda \lambda$ $(7135 \AA+7751 \AA) / \lambda 5192 \AA$ published in Keenan et al. (1988). As shown by Izotov et al. (2006) and Nicholls et al. (2012), the widely used exponential law of [O III] especially suffers from the approximations of temperature-independent collision strengths.

\section{The electron density $n_{\mathrm{e}}$}

To derive the electron density $n_{\mathrm{e}}$, a pair of lines with nearly the same excitation state is chosen. Both of them have to be metastable. In case of thin media, without collisions of the ions with electrons, the line ratio is given simply by their Einstein coefficients. When density increases, the lines with longer lifetimes are affected by collisional de-excitation first. Towards the high-density limit, the ratios of the lifetimes give the line ratio. Owing to the transition, the line ratio is an indicator for the density between these limits. The major representatives for these diagrams are [S II] $\lambda \lambda 6716 \AA / 6732 \AA$ in the calibration of OF06 and [O II] $\lambda \lambda 3726 \AA / 3729 \AA$ by Pradhan et al. (2006). The latter pair is difficult to observe because of the small distance between the lines. Both ions have ionization energies (from the lower state) close to that of hydrogen. The line pair of [Ar IV] $\lambda \lambda 4711 \AA / 4740 \AA$, calibrated by Stanghellini \& Kaler (1989, hereinafter SK89), is sparsely used. The blue line of the pair is very near to a He I emission. On the other hand, this ionization state fits the often used temperature determination using [O III] well (see above).

The models used here assume thermalization of the electrons. The thermalization of the electrons is a major additional boundary condition in our case. Towards extremely thin environments, as can be found in the intergalactic or intracluster medium, these or similar diagnostic diagrams cannot be applied any longer. The influence of non-Maxwellian $\kappa$ distributions on solar wind plasmas has recently been studied by Nicholls et al. (2013). These are plasmas with long-range pumping of electron energies. The timescales are similar to the collisional relaxation time usually denoted to magnetic fields (Leubner 2002; Livadiotis \& McComas 2009). 
We have compiled new diagrams for the two most frequently used diagrams and for the two rarely used diagrams based on argon. We used the CLOUDY C13 code and the included modern atomic data (Ferland et al. 2013). CLOUDY was chosen because it includes the most sophisticated physics we know of for photoionized astrophysical plasmas. Furthermore, we give empirical fit formulas for direct use by observers and for implementation into data reduction recipes.

\section{Setup and calculations}

The three latest versions of the photoionization code CLOUDY, namely C08, C10, and C13, have been used (Ferland et al. 1998, 2013) for our calculations. CLOUDY gives a very sophisticated framework that contains a large set of physical interactions in plasmas with mixed chemistry. Even dust particles are included. The latter are important for charge transfer (van Hoof et al. 2000) and allow molecular $\mathrm{H}_{2}$ and neutral $\mathrm{H}$ I gas to survive in the ionized regions of planetary nebulae (PNe; Aleman \& Gruenwald 2004). Including all those processes is essential for using the diagrams by observers.

In this setup, the temperature or the density (or both of them) are fixed in a thin gas slab, illuminated by a hot OB star at a distance of $0.1 \mathrm{pc}$. For simple calculations for diagnostic diagrams of individual isolated ions in an electron gas, this setup with a pair of fixed values would be sufficient. However, a full physical description with fluorescence and forbidden-line cooling requires complete equilibrium between heating and cooling. Only then is it fully applicable to analyzing observations of nebulae, therefore the stellar temperature or the luminosity are varied until this equilibrium is found. We find no major variations, except for [O III] we find differences worth to be included properly. We attribute this to the effects of the Bowen fluorescence. The whole parameter space is scanned using the vary function in C13. Therefore up to about 100 models are calculated for every data point along the curve. A dedicated program was written to select the appropriate results. An example set of input files is described in the appendix of Proxauf (2013) and can be obtained electronically from the authors. At plasma temperatures above $26000 \mathrm{~K}$, no physically reasonable equilibrium for thermally dominated radiation processes by photoionization is possible. Forbidden-line cooling dominates here. Thus we conclude that these temperatures cannot be reached in normal photoionized regions without other processes (e.g., shocks), therefore, our investigations end at slightly lower temperatures than do those shown in literature before.

\section{Results}

\subsection{Electron temperature}

To derive electron temperatures, the line set [O III] $\lambda \lambda(4958 \AA+$ $5007 \AA) / \lambda 4363 \AA$ is mostly used from the two lower transitions ${ }^{1} D_{2} \rightarrow{ }^{3} P_{2}(2.513 \rightarrow 0.038 \mathrm{eV})$ and ${ }^{1} D_{2} \rightarrow{ }^{3} P_{1}(2.513 \rightarrow$ $0.014 \mathrm{eV})$ and the upper transition ${ }^{1} S_{0} \rightarrow{ }^{1} D_{2}(5.354 \rightarrow$ $2.513 \mathrm{eV}$ ) is mostly used. This method is applicable up to electron densities of a few $10^{4} \mathrm{~cm}^{-3}$. The exponential formula in OF06 was derived using just the basic thermodynamic system of these lines. Pumping via the Bowen fluorescence causes additional populations of the upper level at higher temperatures. Moreover, new atomic data is available. Using the atomic data of Palay et al. (2012), already Nicholls et al. (2013) point out that the exponential formula systematically overestimates the

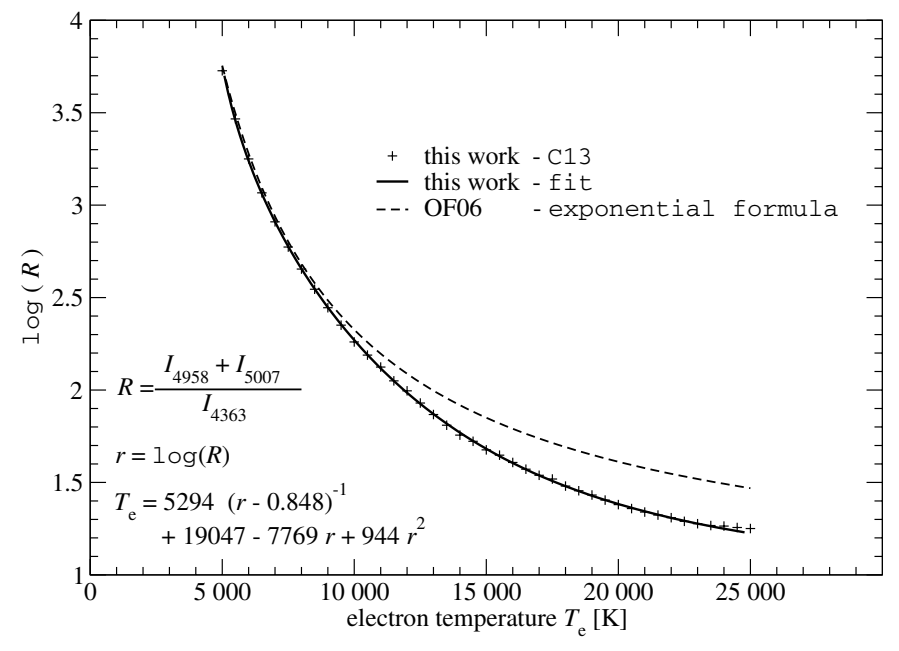

Fig. 1. Diagnostic diagram for the electron temperature using the [O III] lines.

temperature. In Eq. (1) our conversion into temperature for the observed line ratio $R$ is given. Vice versa, since the observers have line ratios $R$ and determine the electron temperature from them, we obtain the given empirical fit (Fig. 1). To reach a perfect correlation, a polynomial correction is applied to the residuals. The fit is valid from 5000 to $24000 \mathrm{~K}$. Starting already at $T_{\mathrm{e}} \approx 10^{4} \mathrm{~K}$, strong deviations from the widely used exponential formula are evident.

$r=\log (R)$

$T_{\mathrm{e}}[\mathrm{K}]=5294(r-0.848)^{-1}+19047-7769 r+944 r^{2}$.

A widely unnoted determination is the one of Keenan et al. (1988) for [Ar III] $\lambda \lambda(7135 \AA+7751 \AA) / \lambda 5192 \AA$ from the two lower transitions ${ }^{1} D_{2} \rightarrow{ }^{3} P_{2}(1.737 \rightarrow 0.000 \mathrm{eV})$ and ${ }^{1} D_{2} \rightarrow{ }^{3} P_{1}(1.737 \rightarrow 0.138 \mathrm{eV})$ and the upper transition ${ }^{1} S_{0} \rightarrow{ }^{1} D_{2}(4.124 \rightarrow 1.737 \mathrm{eV})$. One reason for such rare use is certainly the red wavelength of the lower transitions. Older spectrographs did not work well in that domain. A second reason might be that only a plot, but no table or formula, was given in the original paper. On the other hand, compared to [O III], the lower energies of the individual levels make in particular the upper transition stronger. Additionally, it is not influenced by fluorescence mechanisms:

$r=\log (R)$

$T_{\mathrm{e}}[\mathrm{K}]=11960(r-1.02)^{-1}$.

Current atomic data give a temperature about $10 \%$ below the one given by the calibration of Keenan et al. (1988) (see Fig. 2).

\subsection{Electron density}

To the best of our knowledge, the most often used diagnostic diagram for $n_{\mathrm{e}}$ is that of [S II] $\lambda \lambda 6716 \AA / 6732 \AA$ with the transitions ${ }^{2} D_{5 / 2}^{0} \rightarrow{ }^{4} S_{3 / 2}^{0}(1.846 \rightarrow 0.000 \mathrm{eV})$ and ${ }^{2} D_{3 / 2}^{0} \rightarrow{ }^{4} S_{3 / 2}^{0}$ $(1.842 \rightarrow 0.000 \mathrm{eV})$. The calibration published by OF06 is identical to our results in the setup using $\mathrm{C} 08$. The new atomic data in C10 and C13 lead to systematically 20-22\% lower electron densities. The curve can be fitted fairly well with an arctan function. After the inversion (required for the observer), this leads to 
B. Proxauf et al.: Upgrading electron temperature and electron density diagnostic diagrams of forbidden line emission $(R N)$

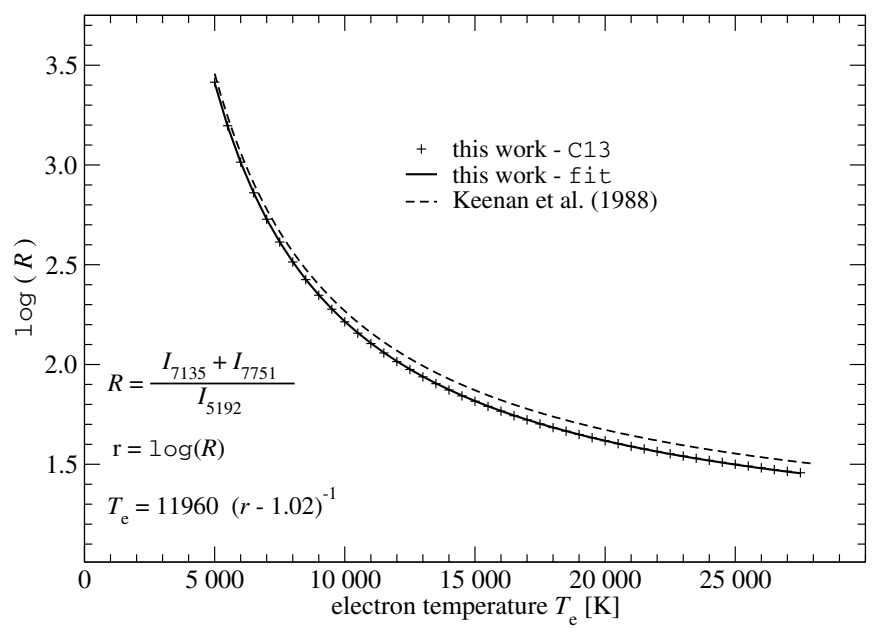

Fig. 2. Temperature derivation using the [Ar III] lines. The new calibration is about $10 \%$ below the older ones throughout the whole range. The fit overlays the data completely.

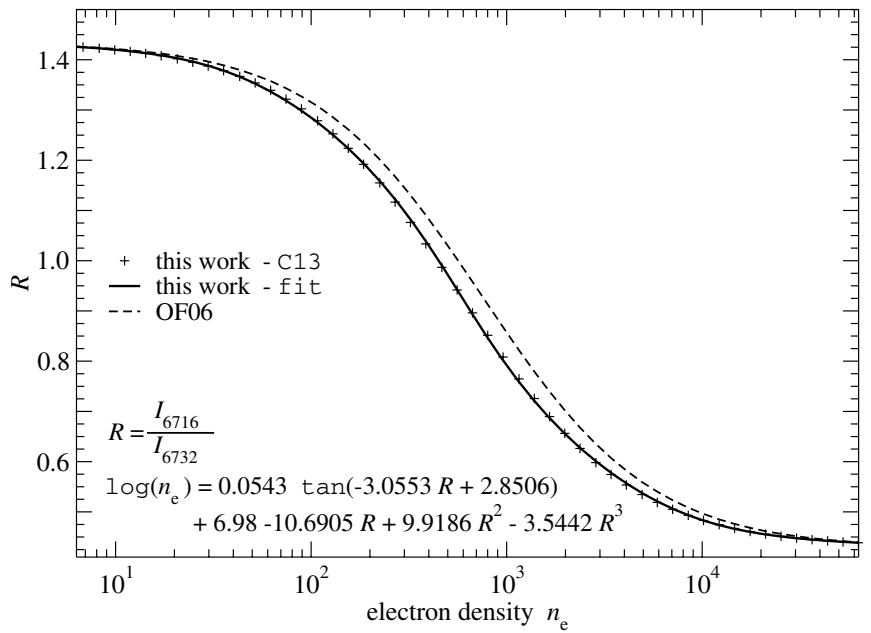

Fig. 3. Electron density derived by the forbidden lines of [S II] at an electron temperature $T_{\mathrm{e}}=10000 \mathrm{~K}$. During the complete, easily used range of $40 \leq n_{\mathrm{e}} \leq 10^{4} \mathrm{~cm}^{-3}$, the result is $20 \%$ to $22 \%$ lower than the widely used OF06 calibration.

the tan function of the resulting function below. To achieve perfect transitions at the lower and upper ends, a polynomial correction was required (Eq. (3)). Same as for the electron temperature, we calculated an empirical fit (see Fig. 3) for $T_{\mathrm{e}}=10000 \mathrm{~K}$ :

$$
\begin{aligned}
\log \left(n_{\mathrm{e}}\left[\mathrm{cm}^{-3}\right]\right)= & 0.0543 \tan (-3.0553 R+2.8506) \\
& +6.98-10.6905 R \\
& +9.9186 R^{2}-3.5442 R^{3} .
\end{aligned}
$$

We know that most nebulae have a co-existence of cold dense clumps, embedded in hotter surrounding thin gas. Thus the analysis to derive the pair $\left(T_{\mathrm{e}}, n_{\mathrm{e}}\right)$ (e.g., for further investigations like abundances) has to take care to obtain information for the same regions within the gas. In most cases these different populations are not spatially resolved in the spectra. Since the ionization energy of $\mathrm{S} \mathrm{I} \rightarrow \mathrm{S}$ II $(12.20 \mathrm{eV})$ is below that of hydrogen, the states can be easily reached by thermal collisional excitation or by absorption of Lyman photons of recombining hydrogen. The next ionization state $\mathrm{S} \mathrm{II} \rightarrow \mathrm{S}$ III requires $23.3 \mathrm{eV}$. This can be reached in regions that are optically thin for UV photons or through photons only developing from $\mathrm{He}$ recombination, so we have to expect the majority of the $[\mathrm{S} \mathrm{II}]$ lines originates in denser clumps or on the outskirts of nebulae.

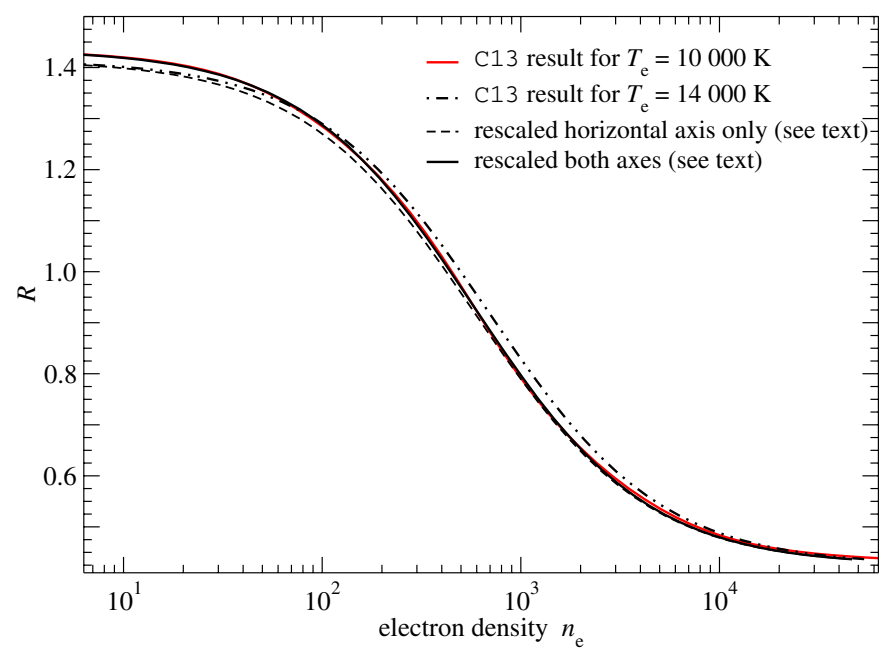

Fig. 4. Scaling of the $[\mathrm{S}$ II $]$ density for other temperatures. A comparison of the diagram at $T_{\mathrm{e}}=10000 \mathrm{~K}$ (red) and $T_{\mathrm{e}}=14000 \mathrm{~K}$ (dashdotted line) is shown. The rescale of the horizontal axis by $n_{\mathrm{e}}\left(10^{4} / T_{\mathrm{e}}\right)^{1 / 2}$ (dashed line) is not perfect. An additional scale of the vertical axis results in nearly undistinguishable curves (solid black line). (This figure is available in color in electronic form.)

At other electron temperatures, the curves are shifted slightly, but have nearly the same shape. Thus SK89 argued that the effect can be ignored. For higher quality of modern spectra, however, small corrections should be applied. OF06 suggest to rescale the horizontal axis by $n_{\mathrm{e}}\left(10^{4} / T_{\mathrm{e}}\right)^{1 / 2}$. As we tested here (Fig. 4), this does not lead to a good result at the low-density regime. We applied an additional linear rescale of the vertical axis by the difference between the low-density limit $R_{\text {low }}^{[\mathrm{S} \mathrm{I}]}$ and the temperature-independent high-density limit $R_{\text {high }}^{[\mathrm{SII}}$. This makes the curves perfectly overlaid.

A full set of limits for electron temperatures $5000 \leq T_{\mathrm{e}} \leq$ $26000 \mathrm{~K}$ is calculated. It can be described by a quadratic approximation and thus give us the detailed rescale recipe by

$$
\begin{aligned}
& R_{\text {low }}^{[\mathrm{S} \mathrm{II}]}\left(T_{\mathrm{e}}\right)=1.496-0.07442\left(\frac{T_{\mathrm{e}}[\mathrm{K}]}{10^{4}}\right)+0.01225\left(\frac{T_{\mathrm{e}}[\mathrm{K}]}{10^{4}}\right)^{2} \\
& R_{\text {high }}^{[\mathrm{S} \mathrm{II}]}=0.436 \\
& R_{\text {obs }}^{[\mathrm{S} \mathrm{II}]}\left(T_{\mathrm{e}}\right) \rightarrow R_{\mathrm{re}-\text { scaled }}^{[\mathrm{S} \mathrm{II}} \\
& R_{\text {re-scaled }}^{[\mathrm{S} \mathrm{II}]}=\frac{R_{\text {low }}^{[\mathrm{SII}]}(10000 K)-R_{\text {high }}^{[\mathrm{SII}]}}{R_{\text {low }}^{[\mathrm{SII}]}\left(T_{\mathrm{e}}\right)-R_{\text {high }}^{[\mathrm{SII}]}}\left(R_{\mathrm{obs}}^{[\mathrm{S} \mathrm{II}]}\left(T_{\mathrm{e}}\right)-R_{\text {high }}^{[\mathrm{S} \mathrm{II}]}\right)+R_{\mathrm{high}}^{[\mathrm{S} \mathrm{II}]} \\
& \rightarrow n_{\mathrm{e}}(10000 K)
\end{aligned}
$$

$n_{\mathrm{e}}\left(T_{\mathrm{e}}\right)=n_{\mathrm{e}}(10000 K)\left(\frac{10000}{T_{\mathrm{e}}}\right)^{-\frac{1}{2}}$.

SK89 presented two different calibrations for the electron density using the [ArIV] $\lambda \lambda 4711 \AA / 4740 \AA$ lines with the transitions ${ }^{2} D_{5 / 2}^{0} \rightarrow{ }^{4} S_{3 / 2}^{0}(2.631 \rightarrow 0.000 \mathrm{eV})$ and ${ }^{2} D_{3 / 2}^{0} \rightarrow{ }^{4} S_{3 / 2}^{0}$ $(2.615 \rightarrow 0.000 \mathrm{eV})$. They used the atomic data sets of Mendoza (1983) and Zeippen et al. (1987). Our new results are presented in Fig. 5. The differences for the derived $n_{\mathrm{e}}$ are fairly high (up to a factor of five). Derived in the same way as above, the fit function is

$$
\begin{aligned}
\log \left(n_{\mathrm{e}}\left[\mathrm{cm}^{-3}\right]\right)= & 0.0846 \tan (-2.4153 R+4.9367) \\
& +5.17-3.16118 R \\
& +2.7206 R^{2}-1.221 R^{3}
\end{aligned}
$$




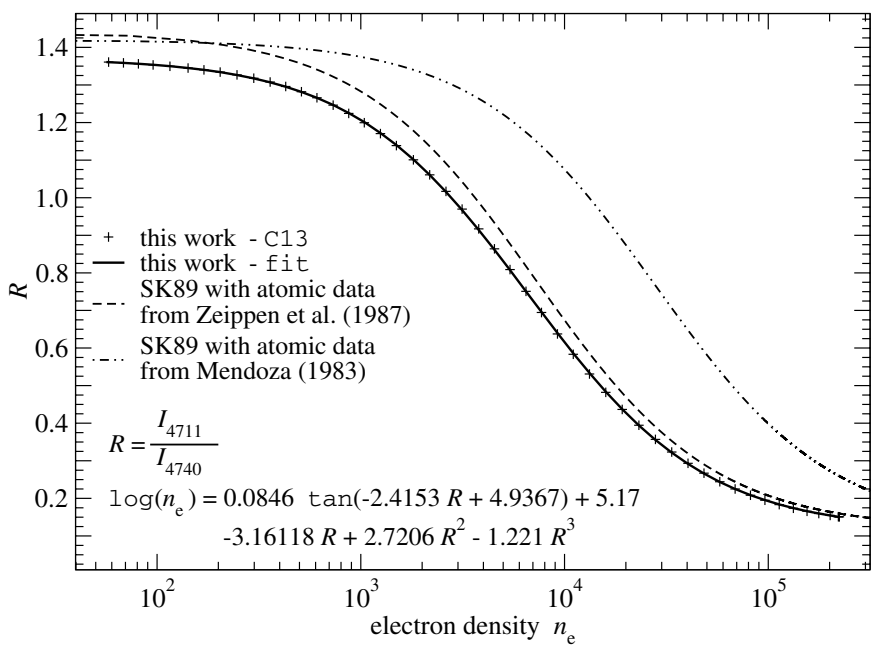

Fig. 5. New diagnostic diagram for [ArIV]. For comparison, the two sets of SK89 are shown.

The ionization energies for ArI $\rightarrow$ ArII, Ar II $\rightarrow$ Ar III, and Ar III $\rightarrow$ Ar IV are $15.76 \mathrm{eV}, 27.63 \mathrm{eV}$, and $40.73 \mathrm{eV}$, respectively. These levels cannot be reached thermodynamically (equilibrium according to Saha's equation) by the typical electron temperatures in $\mathrm{H}$ II regions or PNe. Thus they originate in thin hot regions of the plasma, which are optically thin for UV photons. This makes the $n_{\mathrm{e}}$ ([ArIV]) more suitable for combining with a temperature determination by [O III] lines, compared to $n_{\mathrm{e}}([\mathrm{S}$ II $])$.

For purpose of scaling the low density limits $R_{\text {low }}^{[\mathrm{ArIV}]}$ are calculated for the electron temperature range $8000 \stackrel{\leq}{\leq} T_{\mathrm{e}} \leq$ $26000 \mathrm{~K}$. At lower temperatures, the lines abruptly vanish owing to their required high excitation energy. The recipe follows the same way as for [S II]:

$R_{\text {low }}^{[\mathrm{Ar} \mathrm{IV}]}\left(T_{\mathrm{e}}\right)=1.4663-0.10806\left(\frac{T_{\mathrm{e}}[\mathrm{K}]}{10^{4}}\right)+0.01266\left(\frac{T_{\mathrm{e}}[\mathrm{K}]}{10^{4}}\right)^{2}$

$R_{\text {high }}^{[\text {Ar IV }]}=0.120$.

\section{Conclusions}

Using the current state-of-the-art code and modern atomic data, significant differences in the given diagnostic diagrams are found compared to older studies. Thus we encourage the observers to use these new determinations for interpreting their data. Moreover, we would like to draw the attention of observers to the use of ions with comparable excitation states for deriving $n_{\mathrm{e}}$ and $T_{\mathrm{e}}$. Owing to the observational constraints, [S II] or [O II] densities are often combined with [O III] electron temperatures. One may draw wrong conclusions by looking at different plasmas. The cold, dense clumps, which are embedded in a hot thin gas, dominate the radiation of the [S II] lines. But they are small and thus observationally not resolved. The hot thin gas around the clumps is the origin of the [O III] lines. This is shown well in the nonlinear correlations of $T_{\mathrm{e}}$ found in data from different ionization potentials (Copetti \& Writzl 2002; Izotov et al. 2006). Modern spectrographs (e.g., X-Shooter at ESO) take a very wide range of wavelengths with a single shot at high resolutions, so more sets of lines should be investigated to improve accuracy and to widen the range of usable density and temperature parameter space.

Acknowledgements. S.Ö. is supported by the Austrian Fonds zur Wissenschaftlichen Forschung, FWF doctoral school project W1227.

\section{References}

Aleman, I., \& Gruenwald, R. 2004, ApJ, 607, 865

Copetti, M. V. F., \& Writzl, B. C. 2002, A\&A, 382, 282

Ferland, G. J., Korista, K. T., Verner, D. A., et al. 1998, PASP, 110, 761

Ferland, G. J., Porter, R. L., van Hoof, P. A. M., et al. 2013, Rev. Mex. Astron. Astrofis., 49, 137

Izotov, Y. I., Stasińska, G., Meynet, G., Guseva, N. G., \& Thuan, T. X. 2006, A\&A, 448, 955

Keenan, F. P., Johnson, C. T., \& Kingston A. E. 1988, A\&A, 202,253

Leubner, M. P. 2002, Ap\&SS, 282, 573

Livadiotis, G., \& McComas, D. J. 2009, J. Geophys. Res., 114, 11105

Mendoza, C. 1983, IAU Symp., 103, 245

Nicholls, D. C., Dopita, M. A., \& Sutherland, R. S. 2012, ApJ, 752, 148

Nicholls, D. C., Dopita, M. A., Sutherland, R. S., Kewley, L. J., \& Palay, E. 2013, ApJS, 207, 21

Osterbrock, D. E., \& Ferland, G. J. 2006, Astrophysics of gaseous Nebulae and active Galactic Nuclei (CA: Sausalito: University Science Books)

Palay, E., Nahar, S. N., Pradhan, A. K., \& Eissner, W. 2012, MNRAS, 423, L35

Pradhan, A. K., Montenegro, M. Nahar, S. N., \& Eissner, W. 2006, MNRAS, 366, L6

Proxauf, B. 2013, in Upgrading diagnostic diagrams of forbidden line emission: The influence of modern atomic data, BSc thesis, University Innsbruck, 24 Stanghellini, L., \& Kaler, J.B. 1989, ApJ, 343, 811

van Hoof, P. A. M., Van de Steene, G. C., Beintema, D. A., et al. 2000, ApJ, 532, 384

Zeippen, C.J., Butler, K., \& Le Bourlot, J. 1987, Astr. Ap., 188, 251 